\title{
Avaliação prospectiva da qualidade de vida em uma coorte de pacientes com artrite reumatoide inicial
}

\author{
Licia Maria Henrique da Mota ${ }^{1}$, Ieda Maria Magalhães Laurindo 2 , Leopoldo Luiz dos Santos Neto ${ }^{3}$
}

\begin{abstract}
RESUMO
Introdução: Poucos estudos avaliaram, de forma prospectiva, os instrumentos de aferição de qualidade de vida, tanto genéricos quanto específicos, em pacientes com artrite reumatoide (AR) inicial. Objetivo: O objetivo deste trabalho foi caracterizar uma população de pacientes com AR inicial (menos de 12 meses de sintomas da doença no momento do diagnóstico) acompanhada prospectivamente quanto ao padrão de respostas aos questionários de qualidade de vida Health Assessment Questionnaire (HAQ) e o Medical Outcomes Study SF-36 Health Survey (SF - 36). Pacientes e métodos: Foram avaliados 40 pacientes com diagnóstico de AR inicial no momento do diagnóstico, acompanhados prospectivamente por três anos, em uso de esquema terapêutico padronizado. Registrados os dados demográficos e clínicos e aplicados os questionários HAQ e SF-36 na avaliação inicial e aos 3, 6, 12, 18, 24 e 36 meses de acompanhamento. Comparações feitas pelo teste $t$ de Student, $t$ pareado e Wilcoxon (nível de significância de 5\%). Resultados: A idade média foi de 45 anos e predominou o sexo feminino (90\%). A média do escore do HAQ inicial foi 1,89, com declínio progressivo até 0,77 no terceiro ano $(\mathrm{P}<0,0001)$. A maioria dos elementos do SF-36 apresentaram significativa melhora durante os três anos de seguimento, com exceção de estado geral e vitalidade. Conclusão: Nessa população de pacientes com AR inicial no momento do diagnóstico, observou-se alterações importantes impacto na qualidade de vida no momento do diagnóstico, conforme avaliado pelos questionários HAQ e SF-36. O tratamento precoce da AR parece se associar à melhora da qualidade de vida relacionada com a saúde relatada pelo paciente.
\end{abstract}

Palavras-chave: artrite reumatoide inicial, coorte, HAQ, SF-36, qualidade de vida.

\section{INTRODUÇÃO}

A artrite reumatoide (AR), mesmo em sua fase inicial, pode ocasionar considerável impacto na qualidade de vida relacionada com a saúde (HRQoL - do inglês health-related quality of life). ${ }^{1}$ HRQoL é um conceito bastante amplo, que pode ser simplificado como o impacto da saúde sobre uma habilidade funcional de um indivíduo e no bem-estar percebido nos domínios físico, mental e social da vida. ${ }^{2}$

Vários instrumentos têm sido propostos com a finalidade de avaliar a qualidade de vida nos pacientes com AR, detectar alterações no estado de saúde ao longo do tempo, bem como avaliar prognóstico e os riscos e benefícios de determinada intervenção terapêutica, ${ }^{3}$ incluindo tanto instrumentos genéricos, como o SF-36 (Medical Outcomes Study 36-Item Form Health Survey), quanto instrumentos específicos, como o Health Assessment Questionnaire (HAQ).

Poucos estudos avaliaram de forma prospectiva os instrumentos de aferição de qualidade de vida, tanto genéricos quanto específicos, em pacientes com AR inicial.

O objetivo deste trabalho foi caracterizar uma população de pacientes com AR inicial acompanhada prospectivamente quanto ao padrão de respostas aos questionários de qualidade de vida.

Recebido em 21/03/2010. Aprovado, após revisão, em 30/04/2010. Declaramos a inexistência de conflitos de interesse.

Serviço de Reumatologia do Hospital Universitário de Brasília da Universidade de Brasília ${ }^{1,3}$; Serviço de Reumatologia do Hospital das Clínicas da Faculdade de Medicina da Universidade de São Paulo²

1. Médica-reumatologista do Serviço de Reumatologia do Hospital Universitário de Brasília da Universidade de Brasília (UnB), aluna do programa de Pós-graduação (Doutorado) em Ciências Médicas da Faculdade de Medicina da Universidade de Brasília (UnB)

2. Professora Colaboradora do Hospital das Clínicas da Faculdade de Medicina da Universidade de São Paulo (HCFMUSP)

3. Professor Associado de Clínica Médica e do Serviço de Reumatologia do Hospital Universitário de Brasília (HUB) da Universidade de Brasília (UnB)

Endereço para correspondência: Licia Maria Henrique da Mota. SHLS 716/916 Bloco E, salas 501-502. CEP: 70390-904. Centro Médico de Brasília, Asa Sul.

Brasília, DF, Brasil. Tel/fax: 55 (61) 3245-1966. E-mail: licia@unb.br; liciamhmota@yahoo.com.br 


\section{PACIENTES E MÉTODOS}

Foi realizado estudo prospectivo de coorte incidente em que foram avaliados pacientes consecutivos com o diagnóstico de AR inicial, acompanhados de forma regular por 36 meses a partir do diagnóstico. Os pacientes foram avaliados na Clínica de Artrite Reumatoide Inicial do Hospital Universitário de Brasília, Brasil.

Definiu-se AR inicial como a ocorrência de sintomas articulares compatíveis com a doença (dor e edema articulares de padrão inflamatório, acompanhados ou não de rigidez matinal ou de outras manifestações sugestivas de doença articular inflamatória, segundo avaliação por um observador único), com duração superior a 6 semanas e inferior a 12 meses, independente do preenchimento dos critérios classificatórios do American College of Rheumatology (ACR). ${ }^{4}$ Durante o acompanhamento, confirmou-se o diagnóstico de AR em todos os casos.

A pesquisa de fator reumatoide (FR) foi feita utilizando os ensaios "Quanta Lite ${ }^{\mathrm{TM}}$ FR IgA ELISA", "Quanta Lite ${ }^{\mathrm{TM}}$ FR IgG ELISA" e "Quanta Lite ${ }^{\text {TM }}$ FR IgM ELISA" (Inova Diagnostics, CA, EUA), de acordo com o protocolo do fabricante. Foram considerados como pontos de corte, para se considerar o teste positivo, valores superiores a $15 \mathrm{UI} /$ $\mathrm{mL}$ (FR IgM e IgA) e $20 \mathrm{UI} / \mathrm{mL}$ (FR IgG). Anti-CCP foi pesquisado utilizando os ensaios "Quanta Lite ${ }^{\mathrm{TM}} \mathrm{CCP}$ IgG ELISA", "Quanta Lite ${ }^{\mathrm{TM}}$ CCP3 IgG ELISA" e "Quanta Lite ${ }^{\mathrm{TM}}$ CCP3.1 IgG/IgA ELISA" (Inova Diagnostics, CA, EUA), de acordo com o protocolo do fabricante. Os resultados foram expressos em unidades (U), sendo negativo $<20 \mathrm{U}$, positivo fraco de 20-39 U, positivo moderado de 40-59 U e positivo forte $\geq 60 \mathrm{U}$, para todos os ensaios.

Registrou-se também a ocorrência de erosão radiográfica à radiografia convencional na primeira consulta.

Como instrumentos de avaliação do impacto da AR inicial na qualidade de vida dos pacientes foram utilizadas as versões traduzidas e validadas para a língua portuguesa dos questionários $\mathrm{HAQ}^{5} \mathrm{e} \mathrm{SF}-36 .^{6}$

Registrados os dados demográficos e clínicos e aplicados questionários de qualidade de vida na avaliação inicial e aos $3,6,12,18,24$ e 36 meses de acompanhamento. Os pacientes foram vistos sempre pelo mesmo avaliador, em todas as consultas.

Durante todo o acompanhamento, os pacientes receberam o esquema-padrão de tratamento utilizado no serviço, incluindo drogas modificadoras do curso da doença (DMARDs) tradicionais e/ou terapia modificadora da resposta biológica, de acordo com a necessidade.
Empregou-se o teste de normalidade Kolmogorov-Smirnov e a distribuição foi considerada normal se $\mathrm{P}>0,05$. Para a deteç̧ão de diferenças entre duas médias, utilizou-se o teste $t$ de Student ou teste $t$ pareado para as amostras de distribuição normal, e os testes não paramétricos de Wilcoxon ou de Mann-Whitney para aquelas amostras para as quais a hipótese de normalidade foi rejeitada. A significância foi considerada em caso de $\mathrm{P}<0,05$.

O cálculo do tamanho da amostra foi realizado a partir de uma amostra-piloto de 10 pacientes, partindo-se do princípio de que a metodologia estatística utilizada foi uma análise de variância multivariada para medidas repetidas com interação entre os grupos. Considerando-se um nível de significância de $5 \%$, um poder de teste de $80 \%$ e as informações obtidas da amostra-piloto, chegou-se ao tamanho mínimo de amostra de 40 pacientes.

O trabalho foi aprovado pelo Comitê de Ética em Pesquisa da Faculdade de Medicina da Universidade de Brasília (Registro do projeto: CEP-FM 028/2007)

\section{RESULTADOS}

\section{Características da população estudada}

Foram avaliados 40 pacientes com o diagnóstico de AR inicial. Predominou o sexo feminino (36 pacientes, 90\%) e a idade média foi de 45,3 anos (21 a 71). O período médio da duração dos sintomas articulares no momento do diagnóstico foi de 27,2 semanas ( 4 a 48), sendo que 13 pacientes (32,5\%) tinham menos de 12 semanas de sintomas ao diagnóstico. A maioria dos pacientes (92\%) não recebeu tratamento prévio para $\mathrm{AR}$ até o momento da avaliação inicial.

Não houve perda de seguimento de qualquer paciente durante os três anos de duração do estudo.

A Tabela 1 resume as características demográficas, clínicas, sorológicas e radiográficas da população avaliada.

\section{Esquemas terapêuticos}

$\mathrm{Na}$ avaliação inicial, os 40 pacientes vinham em uso de antiinflamatórios não esteroides (AINE) e dois deles (5\%) utilizavam prednisona em dose inferior a $10 \mathrm{mg} / \mathrm{dia}$ (tempo médio de uso de 14,6 $\pm 2,4$ semanas).

Após a avaliação inicial, 37 pacientes (92,5\%) iniciaram o uso de metotrexato (dose inicial de $7,5 \mathrm{mg} / \mathrm{semana}$ ), com suplementação de ácido fólico (5 mg/semana) e hidroxicloroquina (400 $\mathrm{mg} /$ dia) e um indivíduo (2,5\%) iniciou o uso isolado de metotrexato (dose inicial de $7,5 \mathrm{mg} / \mathrm{semana}$ ), com suplementação de ácido fólico (5 mg/semana), por se recusar a receber 


\section{Tabela 1}

Características demográficas, clínicas, sorológicas e radiográficas dos 40 pacientes com diagnóstico de AR inicial em acompanhamento prospectivo no HU

\begin{tabular}{|c|c|c|}
\hline Característica & & n $( \pm)$ ou n (\%) \\
\hline Idade (anos) & & $45,37( \pm 12,01)$ \\
\hline Gênero & $\begin{array}{l}\text { Masculino } \\
\text { Feminino }\end{array}$ & $\begin{array}{l}4(10 \%) \\
36(90 \%)\end{array}$ \\
\hline Grupo étnico & $\begin{array}{l}\text { Branco } \\
\text { Branco/negro } \\
\text { Branco/indígena } \\
\text { Negro } \\
\text { Negro/indígena }\end{array}$ & $\begin{array}{l}14(35 \%) \\
13(32,5 \%) \\
11(27,5 \%) \\
1(2,5 \%) \\
1(2,5 \%)\end{array}$ \\
\hline $\begin{array}{l}\text { Duração dos sintomas antes } \\
\text { do diagnóstico (semanas) }\end{array}$ & & $27,22( \pm 15,59)$ \\
\hline Acometimento articular & $\begin{array}{l}\text { Agudo } \\
\text { Insidioso }\end{array}$ & $\begin{array}{l}32(80 \%) \\
8(20 \%)\end{array}$ \\
\hline Sinovite persistente de mãos & & $32(80 \%)$ \\
\hline $\begin{array}{l}\text { Duração da rigidez } \\
\text { matinal (minutos) }\end{array}$ & & $155,5( \pm 117,97)$ \\
\hline Número de articulações dolorosas & & $20,07( \pm 6,74)$ \\
\hline $\begin{array}{l}\text { Número de articulações } \\
\text { edemaciadas }\end{array}$ & & $14,15( \pm 7,64)$ \\
\hline Nódulos reumatoides & & $5(12,5 \%)$ \\
\hline $\begin{array}{l}\text { Outras manifestações ou } \\
\text { diagnósticos associados }\end{array}$ & $\begin{array}{l}\text { Fadiga } \\
\text { Anemia } \\
\text { Fenômeno de } \\
\text { Raynaud } \\
\text { Vasculite cutânea } \\
\text { Manifestações } \\
\text { pulmonares } \\
\text { Manifestações } \\
\text { oculares } \\
\text { Manifestações } \\
\text { cardíacas } \\
\text { Fibromialgia } \\
\text { Sintomas } \\
\text { depressivos } \\
\text { Síndrome sicca }\end{array}$ & $\begin{array}{l}28(70 \%) \\
7(17,5 \%) \\
4(10 \%) \\
2(5 \%) \\
2(5 \%) \\
2(5 \%) \\
0(0) \\
9(22,5 \%) \\
8(20 \%) \\
7(17,5 \%)\end{array}$ \\
\hline DAS28 & & $6,86( \pm 1,07)$ \\
\hline Erosão radiográfica & & $21(52,5 \%)$ \\
\hline FR (FR IgA, FR IgG e/ou FR IgM) & & $21(52,5 \%)$ \\
\hline $\begin{array}{l}\text { Anti-CCP (CCP2, CCP3 } \\
\text { e/ou CCP3.1) }\end{array}$ & & $21(52,5 \%)$ \\
\hline
\end{tabular}

As variáveis estão representadas em média do valor absoluto ( \pm desvio-padrão) ou $\mathrm{n}(\%)$. FR = fator reumatoide; DAS = Disease activity índex.

hidroxicloroquina. Dois pacientes $(5 \%)$, com manifestações pulmonares, receberam prescrição de ciclofosfamida $\left(0,8\right.$ e $1 \mathrm{~g} / \mathrm{m}^{2}$ de superfície corporal) e metilprednisolona (1 g) em pulsos mensais. Os dois pacientes com vasculite cutânea utilizaram, além de DMARD, prednisona na dose inicial de $40 \mathrm{mg} / \mathrm{dia}$.

Os esquemas terapêuticos utilizados após três anos estão sumarizados na Figura 1.
Após três anos de acompanhamento, oito pacientes (20\%) mantinham o uso da terapêutica inicialmente prescrita - hidroxicloroquina (dose média de $400 \mathrm{mg} / \mathrm{dia}$ ) e metotrexato (dose média de $20,5 \mathrm{mg} / \mathrm{semana})$.

Vinte e três indivíduos (57,5\%) utilizavam um esquema de DMARD não biológico diferente do inicialmente prescrito. Um paciente fazia uso de metotrexato $(15 \mathrm{mg} / \mathrm{semana})$, hidroxicloroquina (400 mg/dia) e leflunomida (20 mg/dia); seis outros estavam usando metotrexato (dose média de $18,5 \mathrm{mg} / \mathrm{semana}$ ) e leflunomida (20 mg/dia); três utilizavam metotrexato (dose média de 12,5 mg/semana), hidroxicloroquina (400 mg/dia) e sulfassalazina (dose média de $1 \mathrm{~g} /$ dia); nove em monoterapia com leflunomida (20 mg/dia) e um paciente em uso de leflunomida (20 mg/dia) e ciclosporina (100 mg/dia).

\section{Questionários de qualidade de vida}

A diferença entre os valores final e inicial do escore do HAQ e de alguns domínios do SF-36 apresentou distribuição normal, com exceção dos domínios aspectos emocionais e físicos, saúde mental e vitalidade.

A Tabela 2 e as Figuras 2 e 3 mostram os escores obtidos com a aplicação dos questionários de qualidade de vida HAQ e SF-36 na avaliação basal e após três anos de acompanhamento. O escore do HAQ e da maioria dos domínios do SF-36 (com exceção dos domínios estado geral e vitalidade) apresentou melhora significativa entre a avaliação basal e após três anos.

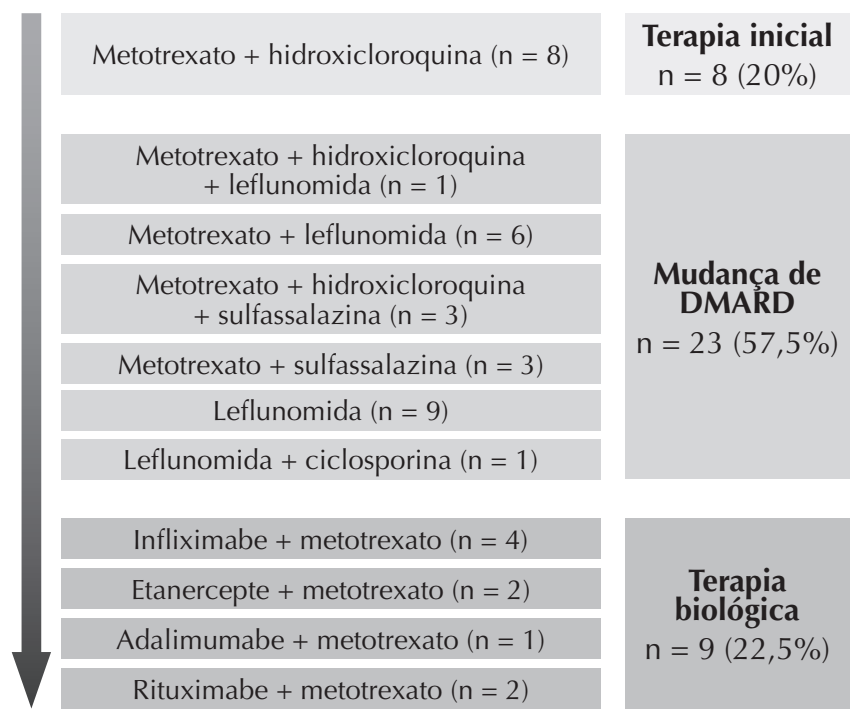

DMARD = droga modificadora do curso da doença.

\section{Figura 1}

Esquemas terapêuticos utilizados pelos 40 pacientes com diagnóstico de AR inicial após três anos de acompanhamento. 
Tabela 2

Escores dos questionários HAQ e SF-36 dos 40 pacientes com diagnóstico de AR inicial em acompanhamento prospectivo no HUB - avaliação basal e após três anos

\begin{tabular}{|c|c|c|c|c|}
\hline \multirow[b]{2}{*}{ Questionários } & \multicolumn{2}{|c|}{ Avaliação Média (desvio-padrão) } & \multirow[b]{2}{*}{ Valor T } & \multirow[b]{2}{*}{ Valor P } \\
\hline & Basal & Três anos & & \\
\hline HAQ & $1,89(0,78)$ & $0,77(0,64)$ & 7,49 & $<0,0001$ \\
\hline \multicolumn{5}{|l|}{ SF-36 (Domínios) } \\
\hline Aspectos sociais & $41,87(34,27)$ & $77,24(26,42)$ & $-5,77$ & $<0,0001$ \\
\hline Dor & $23,00(19,77)$ & $57,51(23,14)$ & $-7,08$ & $<0,0001$ \\
\hline Estado geral & $57,35(25,86)$ & $66,33(23,72)$ & $-1,77$ & 0,08 \\
\hline \multirow[t]{2}{*}{ Capacidade funcional } & $23,50(25,32)$ & $56,28(29,22)$ & $-5,32$ & $<0,0001$ \\
\hline & \multicolumn{2}{|c|}{ Avaliação Mediana (amplitude interquartil) } & & \\
\hline Questionários & Basal & Três anos & Teste de Wilcoxon & Valor P \\
\hline \multicolumn{5}{|l|}{ SF-36 (Domínios) } \\
\hline Saúde mental & $52,00(20,00)$ & $56,00(12,00)$ & 133,00 & 0,03 \\
\hline Aspectos emocionais & $0(0)$ & $66,67(100,00)$ & 139,50 & $<0,0001$ \\
\hline Vitalidade & $50,00(15,50)$ & $50,00(20,00)$ & 14,00 & 0,08 \\
\hline Aspectos físicos & $0(0)$ & $25,00(100,00)$ & 146,50 & $<0,0001$ \\
\hline
\end{tabular}

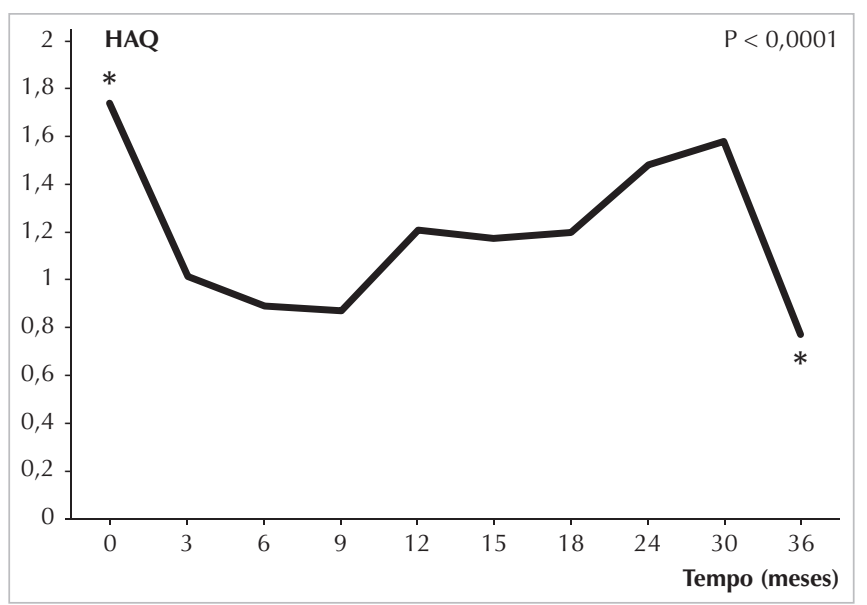

Figura 2

Escore médio do questionário HAQ dos 40 pacientes com diagnóstico de AR inicial em acompanhamento prospectivo no HUB por três anos.

HUB = Hospital Universitário de Brasília; $A R=$ artrite reumatoide; $\mathrm{HAQ}=$ Health Assessment Questionnarie.

\section{DISCUSSÃO}

Muitas coortes têm utilizado o instrumento HAQ, ${ }^{7}$ de modo que é possível fazer comparações entre os resultados obtidos neste estudo com outras populações de pacientes com diagnóstico de AR inicial.
As coortes publicadas demonstram uma ampla variação da média do HAQ na avaliação inicial, mas a média do escore encontrado na maioria delas gira em torno de $1(0,8$ a 1,3$),{ }^{8-11}$ valor inferior ao encontrado em nossa população. Em nossa coorte, a média do escore do HAQ na avaliação inicial foi de 1,9 , caindo para 0,8 após três anos de acompanhamento.

Embora a maioria dos estudos mostre melhora no escore do HAQ ao longo dos primeiros anos após o diagnóstico de AR, há grande variação na taxa de mudança do HAQ. Após dois anos de seguimento, 10\% dos 147 pacientes do estudo de van der Heijde et al. ${ }^{12}$ e $29 \%$ do estudo de Wiles et al. ${ }^{9}$ ainda apresentavam um escore do HAQ maior que 1. Combe et al. ${ }^{11}$ reportaram que $27 \%$ dos 191 pacientes de sua coorte apresentavam HAQ $>1$ após três anos de acompanhamento, e $22 \%$ ainda mantinham essa média após cinco anos. As cifras obtidas na coorte de Young et al. ${ }^{8}$, nos mesmos períodos (três e cinco anos), foram de 34\% e 38\%. Possíveis explicações para as variações de evolução do escore do HAQ incluem diferentes desenhos entre os estudos e influência de outras variáveis, como os efeitos do tratamento e status socioeconômico. ${ }^{13}$

Seis grandes estudos envolvendo pacientes com AR inicial, do Reino Unido - NOAR ${ }^{14}$ e ERAS,$-{ }^{15}$ Suécia, França e Holanda ${ }^{16-19}$, mostram que o comportamento do HAQ apresenta uma "curva em J", observando-se uma queda inicial do escore do HAQ, seguido por um aumento gradual ao longo dos anos. Nesses estudos, a média do escore do HAQ inicial 

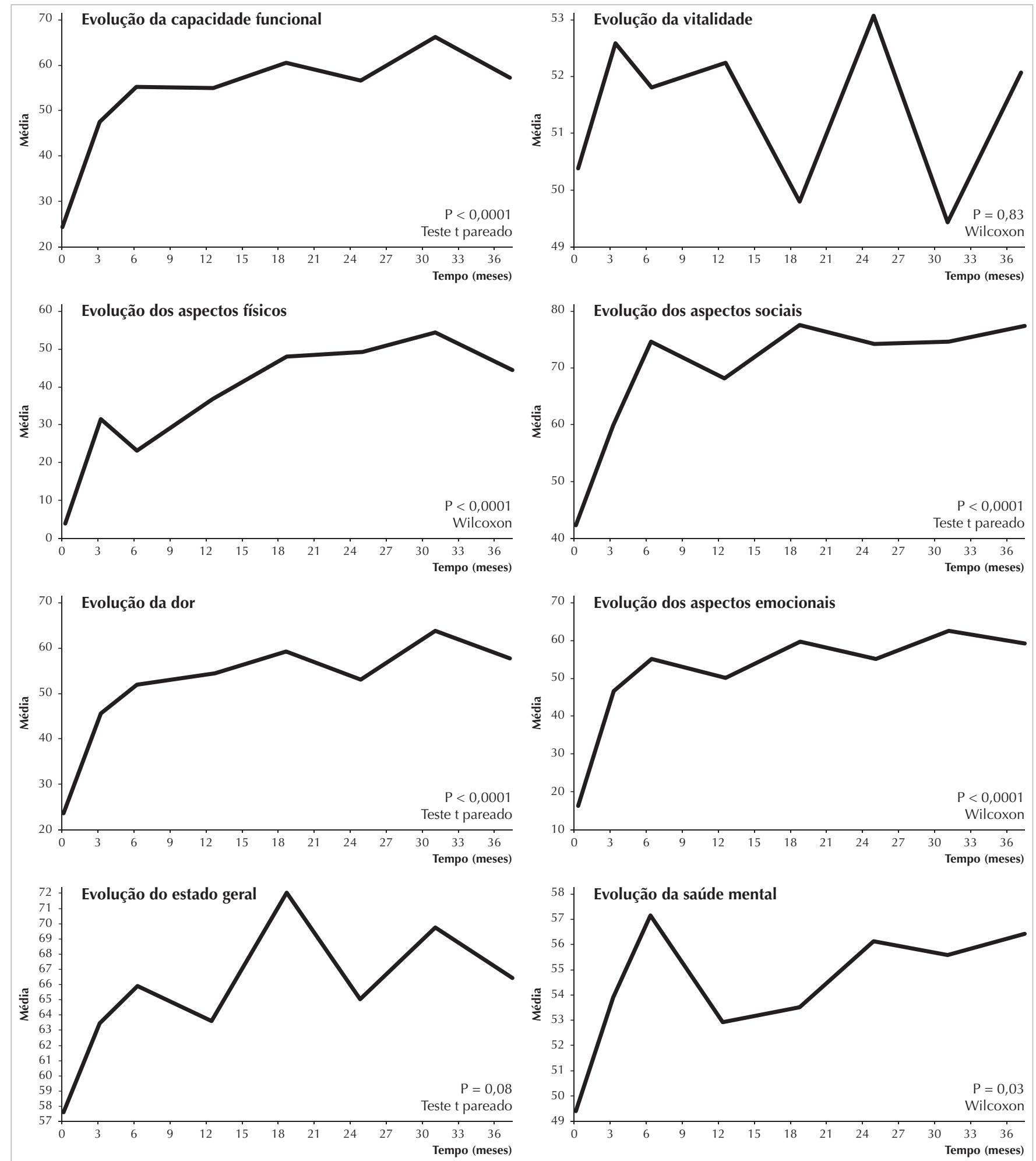

Figura 3

Escores (médias - teste $t$ pareado e medianas - Wilcoxon) dos domínios do questionário SF-36 referentes aos 40 pacientes com diagnóstico de AR inicial em acompanhamento prospectivo no HUB por três anos.

HUB = Hospital Universitário de Brasilia; AR = artrite reumatoide; SF-36 Medical Outcomes Study 36-Item Form Health Survey. 
foi de 0,92 (variando de 0,63 a 1,3). Após três anos, a média caiu para 0,74 , mas subsequentemente aumentou até 0,83 , no quinto ano.

Uma possível explicação para essa "curva em J" é que o paciente com AR tem uma incapacidade considerável antes do início do tratamento. A terapia com AINE e DMARD inicialmente melhora a sinovite e a incapacidade a ela associada. No entanto, a limitação funcional aumenta lenta e progressivamente à medida que o dano articular e outras manifestações da doença progridem..$^{20} \mathrm{Em}$ nossa coorte, a melhora do escore do HAQ foi rápida (grande decréscimo do escore já nos primeiros seis meses de tratamento), havendo piora progressiva (aumento do escore do HAQ) entre o sexto e o trigésimo mês de tratamento. A partir do mês 30, até o final do mês 36, houve mais uma vez melhora progressiva do escore, que atingiu valor médio significativamente inferior ao inicial.

Quanto ao questionário SF-36, os reduzidos valores dos escores dos domínios na avaliação inicial demonstraram grande impacto na qualidade de vida relacionada com a saúde em nossa população de pacientes com AR inicial. Em especial os domínios "limitação por aspectos físicos" e "limitação por aspectos emocionais" obtiveram pontuação muito baixa, demonstrando que, nos pacientes de nossa coorte, são esses os aspectos da qualidade de vida mais prejudicados na avaliação inicial. Os domínios "saúde mental" e "vitalidade" foram os menos comprometidos no momento do diagnóstico.

West e Jonsson relataram os escores do questionário SF-36 em um grupo de 40 pacientes com AR inicial acompanhados por dois anos. Na avaliação inicial, os pacientes apresentaram valores mais baixos (piores) para todos os oito domínios do SF-36 em relação ao grupo-controle. Após dois anos, os pacientes relataram melhora em muitos dos domínios avaliados, incluindo dor e função física. ${ }^{21}$

Comparações com outras coortes são menos factíveis quando utilizado o questionário SF-36, em relação ao HAQ, uma vez que existem poucos estudos prospectivos envolvendo pacientes com AR inicial avaliando o questionário SF-36. Além disso, o fato de o SF-36 ser uma ferramenta genérica, não específica para AR, e portanto sofrer a influência de diversas outras condições, como idade, sexo, status socioeconômico, presença de comorbidades, incluindo sintomas depressivos e fibromialgia, ${ }^{22}$ torna difícil a comparação de escores entre diferentes populações.

Uhlig et al. ${ }^{23} \mathrm{e}$ Talamo et al. ${ }^{24}$ publicaram os dados da análise da HRQol em 1.052 pacientes do Registro de AR de Oslo e 137 pacientes ingleses, respectivamente. Os dois grupos relataram uma performance melhor que a observada em nossa coorte em seis dos domínios do SF-36, com exceção de "estado geral" e "vitalidade", que apresentaram maior pontuação entre os pacientes do presente estudo. Tanto no trabalho norueguês quanto no inglês, no entanto, não foram avaliados pacientes com AR inicial, e sim indivíduos com AR estabelecida (13,8 e 11 anos de evolução da doença, em média, respectivamente) e em uso de DMARD e biológicos, o que torna difícil a comparação com os dados iniciais da nossa coorte.

Neste estudo, todos os domínios avaliados, com exceção do estado geral de saúde e vitalidade, apresentaram melhora significativa ao longo dos três anos de acompanhamento. Os escores finais obtidos em nossa coorte foram semelhantes aos publicados por Wiles et al. ${ }^{25}$ referentes ao quinto ano de acompanhamento de 303 pacientes com diagnóstico de AR inicial da coorte NOAR.

Não há trabalhos anteriores que tenham avaliado, como o nosso, o perfil de evolução dos domínios do SF-36 ao longo do tempo. Em nossa coorte, o diferente padrão apresentado pelos domínios durante os 36 meses de acompanhamento pode refletir impactos diversos da doença e do seu tratamento sobre as características avaliadas, ou ser atribuído à subjetividade das respostas ao questionário utilizado, que sofre a influência de diversos outros aspectos.

A população acompanhada em nossa coorte apresentou grande impacto da AR em sua qualidade de vida, conforme avaliado pelos questionários HAQ e SF-36, no momento do diagnóstico, maior do que em outras coortes anteriormente avaliadas. Em contrapartida, houve uma considerável melhora da incapacidade já nos primeiros meses de acompanhamento, melhora essa que se manteve no final do terceiro ano. $\mathrm{O}$ tratamento precoce da AR pareceu, em nossa coorte, estar associada à melhora da qualidade de vida relacionada à saúde relatada pelo paciente.

\section{CONCLUSÕES}

A população avaliada na coorte deste estudo apresentou um grande impacto da AR em sua qualidade de vida, conforme avaliado pelos questionários HAQ e SF-36, no momento do diagnóstico, maior do que em outras coortes anteriormente avaliadas.

Em uma coorte de pacientes com diagnóstico precoce de AR recebendo tratamento-padrão para a doença, houve melhora significativa da qualidade de vida avaliada pelos questionários HAQ e da maioria dos componentes da saúde física e mental do SF-36, excetuando-se apenas o estado geral de saúde e a vitalidade. $\mathrm{O}$ tratamento precoce da $\mathrm{AR}$ parece associar-se à melhora da qualidade de vida relacionada com a saúde relatada pelo paciente. 


\section{REFERÊNCIAS}

\section{REFERENCES}

1. Kosinski M, Kujawski SC, Martin R, Wanke LA, Buatti MC, Ware JR JE et al. Health-related quality of life in early rheumatoid arthritis: impact of disease and treatment response. Am J Manag Care 2002; 8:231-40.

2. Ward MM. Outcome measurement: health status and quality of life. Curr Opin Rheumatol 2004; 16:96-101.

3. Walker JG, Littlejohn GO. Measuring quality of life in rheumatic conditions. Clin Rheumatol 2007; 26:671-3.

4. Arnett FC, Edworthy SM, Bloch DA, McShane DJ, Fries JF, Cooper NS et al. The American Rheumatism Association 1987 revised criteria for the classification of rheumatoid arthritis. Arthritis Rheum 1988; 31:315-24.

5. Ferraz MB. Tradução para o português e validação do questionário para avaliar a capacidade funcional "Stanford Health Assessment Questionnaire" Doutorado [Tese]. São Paulo: Universidade Federal de São Paulo; Escola Paulista de Medicina, 1990. 
6. Ciconelli RM, Ferraz MB, Santos WS, Meinão I, Quaresma MR. Tradução para a língua portuguesa e validação do questionário genérico de qualidade de vida SF-36 (Brasil SF-36). Rev Bras Reumatol 1999; 39:143-50.

7. Fries JF. New instruments for assessing disability: not quite ready for prime time. Arthritis Rheum 2004; 50:3064-7.

8. Young A, Dixey J, Cox N, Davies P, Emery P, Gallivan S. How does functional disability in early rheumatoid arthritis (RA) affect patients and their lives? Results of 5 years of follow-up in 732 patients from the Early RA Study (ERAS). Rheumatology (Oxford) 2000; 39:603-11.

9. Wiles NJ, Dunn G, Barrett EM, Harrison BJ, Silman AJ, Symmons DP. One year follow up variables predict disability 5 years after presentation with inflammatory polyarthritis with greater accuracy than at baseline. J Rheumatol 2000; 27:2360-6.

10. Eberhardt KB, Fex E. Functional impairment and disability in early heumatoid arthritis - development over 5 years. J Rheumatol 1995; 22:1037-42.

11. Combe B, Cantagrel A, Goupille P, Bozonnat MC, Sibilia J, Eliaou JF. Predictive factors of 5-year health assessment questionnaire disability in early rheumatoid arthritis. J Rheumatol 2003; 30:2344-9.

12. van der Heijde DMFM, van Riel PLCM, van Leeuwen MA, van't Hof MA, van Rijswijk MH, van de Putte LB. Prognostic factors for radiographic damage and physical disability in early rheumatoid arthritis. Br J Rheumatol 1992; 8:519-26.

13. Young A, Wilkinson P, Talamo J, Dixey J, Cox N. Socioeconomic factors in the presentation and outcome of early rheumatoid arthritis. Lessons for the health service? Ann Rheum Dis 2000; 59:794-9.

14. Harrison B, Symmons D. Early inflammatory polyarthritis: results from the Norfolk Arthritis Register with a review of the literature II. Outcome at three years. Rheumatology (Oxford) 2000; 39:939-49.

15. Dixey J, Solymossy C, Young A. Early RA Study, Is it possible to predict radiological damage in early rheumatoid arthritis (RA)? A report on the occurrence, progression, and prognostic factors of radiological erosions over the first 3 years in 866 patients from the Early RA Study (ERAS). J Rheumatol Suppl 2004; 69:48-54.

16. Welsing PM, van Gestel AM, Swinkels HL, Kiemeney LALM, van Riel PLCM. The relationship between disease activity, joint destruction, and functional capacity over the course of rheumatoid arthritis. Arthritis Rheum 2001; 44:2009-17.
17. Wiles N, Dunn G, Barrett E, Silman A, Symmons D. Associations between demographic and disease-related variables and disability over the first five years of inflammatory polyarthritis: a longitudinal analysis using generalized estimating equations. J Clin Epidemiol 2000; 53:988-96.

18. Kobelt G, Jonsson L, Lindgren P, Young A, Eberhardt K. Modeling the progression of rheumatoid arthritis: a two-country model to estimate costs and consequences of rheumatoid arthritis. Arthritis Rheum 2002; 46:2310-9.

19. Drossaers-Bakker KW, de Buck M, van Zeben D, Zwiderman $\mathrm{AH}$, Breedveld FC, Hazes JM. Long-term course and outcome of functional capacity in rheumatoid arhtirits: the effect of disease activity and radiologic damage over time. Arthritis Rheum 1999; 42:1854-60

20. Scott DL, Smith C, Kingsley G. What are the consequences of early rheumatoid arthritis for the individual? Best Pract Res Clin Rheumatol 2005; 19:117-36.

21. West E, Jonsson SW. Health-related quality of life in rheumatoid arthritis in Northern Sweden: a comparison between patients with early RA, patients with medium-term disease and controls, using SF-36. Clin Rheumatol 2005; 24:117-22.

22. Birtane M, Uzunca K, Tastekin N, Tuna H. The evaluation of quality of life in fibromyalgia syndrome: a comparison with RA by using SF-36 Health Survey. Clin Rheumatol 2007; 26:679-84.

23. Uhlig T, Loge JH, Kristiansen IS, Kvien TK. Quantification of reduced health-related quality of life in patients with rheumatoid arthritis compared to the general population. J Rheumatol 2007; 34:1241-7.

24. Talamo J, Frater A, Gallivan S, Young A. Use of short form 36 (SF-36) for health status measurement in rheumatoid arthritis. B J Rheumatol 1997; 36:4639.

25. Wiles NJ, Scott DG, Barret EM, Merry P, Arie E, Gaffney K et al. Benchmarking: the five year outcome of rheumatoid arthritis assessed using a pain score, the Health Assessment Questionnaire, and the Short-Form-36 (SF-36) in a commuity and a clinic based sample. Ann Rheum Dis 2001; 60:956-61. 